\title{
Repercusión del conocimiento previo matemático al aprender física en la educación diversificada y en la educación universitaria inicial
}

\author{
Kenneth Castillo-Rodriguez ${ }^{1} \&$ Alejandro Moya-Segura² \\ 1. Licenciado en Enseñanza de las Ciencias, Bachiller en Biología Tropical, Universidad Nacional, Coordinador del \\ Programa de Capacitación Permanente en Didáctica de las Ciencias Experimentales, Universidad Estatal a Distancia, \\ Costa Rica.Correo: kecastillo@uned.ac.cr \\ 2. Licenciado en Enseñanza de las Ciencias, Bachiller en Biología Tropical, Universidad Nacional, Profesor de Biología \\ y Física en los Colegios Marista y Saint Francis, Costa Rica. Correo: aemscr@gmail.com
}

Recibido: 31 de octubre, 2016

Aceptado: 08 de diciembre, 2016

\begin{abstract}
RESUMEN
La presente investigación responde a la interrogante: ¿de qué manera repercute el conocimiento previo matemático a la hora de aprender física, en Educación Diversificada y en la educación universitaria inicial?. La literatura consultada muestra que efectivamente, los estudiantes graduados del sistema educativo formal, deben contar con una serie de conocimientos básicos que les permita abordar el siguiente nivel; la matemática y la física no escapan de esa realidad. La metodología utilizada fue secuencial, en la primera parte se realizó una entrevista a profundidad, aplicada a 10 especialistas. Los instrumentos fueron analizados de manera descriptiva, por medio de matrices y triangulaciones. Como segunda parte, con base a este análisis se crearon cuestionarios, que se aplicaron a 22 profesores de Física de secundaria de la región de Alajuela, a 22 profesores de universidades estatales, a 16 estudiantes del curso de Física General de la Universidad Técnica Nacional (UTN) y a 200 estudiantes de secundaria; los datos fueron analizados de manera conjunta entre las poblaciones por medio de matrices. De acuerdo con las entrevistas se obtuvo que es necesario un cuerpo de conocimientos matemáticos previos, que repercuten en el aprendizaje de la Física desde dos dimensiones: a nivel del pensamiento y de los procedimientos matemáticos. Además, hay metacognición por parte de los estudiantes, en el sentido de que la Matemática es necesaria para aprender Física. De tal manera que la principal recomendación de este estudio es articular todos los planes de estudio desde educación diversificada hasta universidad, y respetar el proceso de construcción del conocimiento.
\end{abstract}

Palabras claves: Conocimientos previos, Física, Matemática, Aprendizaje significativo, Repercusión.

\begin{abstract}
ABSTRAC
This research answers the question: how will impact the mathematical prior knowledge when learning significantly physics in diversified education and initial college? The literature shows that indeed, graduates of some formal education, should have a basic knowledge that allows them to address the next level; mathematics and physics do not escape that reality. The methodology used for data collection was an in-depth interview applied to 10 specialists, the instruments were analyzed qualitatively, using matrices and triangulations. Questionnaires were applied to 22 high school physics teachers in the region of Alajuela, 22 teachers of state universities, 16 students of the course of General Physics on National Technical University (UTN) and 200 high school students too. Data were analyzed qualitatively and quantitatively. It was found that a body of previous mathematical knowledge which affects the learning of physics is necessary from two dimensions: a level of thought and mathematical procedures, plus there metacognition by the students, in the sense that Mathematics is necessary to learn physics. Therefore, it is recommended to articulate all diversified curricula from education to college, and respect the process of knowledge construction.
\end{abstract}

Key words: Physics, Mathematics, Meaningful learning, Impact, Metacognition.

\section{Introducción}

Las dificultades en el desempeño académico en las tres ciencias: física, química y biología, reflejan la necesidad de indagar cuáles factores repercuten para su aprendizaje significativo; en el caso del presente estudio se buscó 
comprender si existe repercusión del nivel de conocimiento matemático al aprender física en la educación diversificada y en la educación universitaria inicial.

Trabajos previos en países como Italia, Francia y Taiwán han tratado de encontrar el por qué la mayor parte de la población estudiantil fracasa en el primer curso de física en la universidad; aunque se han establecido explicaciones, son insatisfactorias debido a que se han tomado causas aisladas (Chang \& Bell, 2002, p. 82). Por su parte, otros estudios realizados como parte del mejoramiento de los cursos de física básica en Argentina (1993) (Salinas, Cudmani y Pesas, 1996, p.211) habían detectado el uso generalizado del razonamiento no científico para dar una respuesta a situaciones conceptuales básicas en física.

En este mismo sentido, Owen, Dickson, Stanisstrest y Boyes (2008) han encontrado que los estudiantes ingresan a secundaria con una actitud positiva hacia la ciencia y en el transcurso de esos años, su actitud hacia esta área del conocimiento termina siendo negativa. En el estudio de estos mismos autores, los estudiantes catalogaron la Física como aburrida, irrelevante y difícil. Y llegaron a recomendar un cambio en el currículo de Física, de manera que se tomen en cuentan conceptos que pueden ser relevantes para el alumnado (Owen et al., 2008, p.120).

La Facultad de Ingenierías de la Universidad Nacional de la Plata, en Argentina, inició en el 2002 un rediseño de los planes de estudio y de los cursos. En los grupos donde se aplicaron los nuevos programas, hubo una mayor comprensión de los conceptos básicos de la Física. Sin embargo, los resultados también arrojaron que entre la matemática y la física se deben unificar nomenclaturas que pueden interferir en la relación de nuevos conocimientos en física (Rossana, Di Domenicantonio, Prodanoff, Tolosa \& Guarepi, 2006).

De acuerdo con el contexto costarricense, se puede mencionar, existe dificultad en el aprendizaje de la Física, no solo a nivel de educación diversificada, sino también en los cursos introductorios de física en la universidad (Alfaro \& Villegas, 2010).
Por ejemplo en el tercer informe del estado de la educación científica en Costa Rica del año 2010, se establece que ha habido un detrimento de dicha educación, a partir de que en la década de los años ochenta se propusiera cambiar la distribución de lecciones dedicadas a las asignaturas científicas (cuatro lecciones para física y química en décimo año y cuatro para biología en undécimo año, más cinco lecciones de una optativa, a pasar a un sistema de tres lecciones por disciplina a la semana). Luego han surgido diversos proyectos por parte de la Universidad Nacional (UNA) y la Universidad de Costa Rica (UCR), tendientes a mejorar la educación científica en Costa Rica, pero los esfuerzos (entre ellos los proyectos de olimpiadas: "Olimpiada Costarricense de Física (OLCOFI)”, "Olimpiada Costarricense e Internacionales de Química (OLCOQUIM)" y “Olimpiada Costarricense de Ciencias Biológicas (OLICOCIBI)") no han sido capaces de resolver los problemas de desempeño en las tres ciencias y esto se reflejan en los cursos introductorios universitarios de Física, Química y Biología (Alfaro \& Villegas, 2010).

Otro ejemplo son las pruebas PISA aplicadas en el país en el año 2012 para analizar la alfabetización matemática y la alfabetización científica. Estas denotaron un desempeño bajo en dichas competencias (Román y Vargas, 2015)

Malas experiencias en el área matemática, y en las disciplinas que se apoyan en el conocimiento matemático y la poca oferta en el área técnico - científica en educación superior, generan un estancamiento en el área de ciencias e ingenierías, tan necesarias actualmente para que el país de un salto positivo en su competitividad. (Román \& Vargas, 2015)

A pesar de estos indicios, hoy no se ha sistematizado dicha información, ni tampoco se ha investigado o encontrado las posibles causas por las cuales los estudiantes se les dificulta el aprendizaje en los cursos iniciales de física universitarios.

\section{Metodología}

El estudio se acoge bajo la base filosófica del pragmatismo como paradigma, esta 
investigación se encuentra sustentada dentro de un enfoque mixto de investigación (Seas, 2015). Los instrumentos que se aplicaron durante la primera fase cualitativa a los especialistas fue: la técnica de entrevista a profundidad, semiestructurada (Hernández, Fernández \& Baptista, 2008). En la segunda fase cuantitativa los cuestionarios para los estudiantes de secundaria, y universidad, fueron elaborados a partir de la información recolectada en las entrevistas a especialistas. Los cuestionarios hechos para estudiantes de secundaria, a docentes de colegio y de cursos de física universitaria fueron diseñados con ítems acompañados de escalas Likert. En el caso de los colegiales se trató de identificar la percepción que tienen acerca de la repercusión del conocimiento matemático a la hora de aprender física.

En el caso de docentes de secundaria y universidad se hicieron algunas variaciones del cuestionario anterior y la mayoría de ítems iban relacionadas, al grado de concordancia que se tiene con respecto a frases extraídas del análisis realizado a las entrevistas de los especialistas. Dichas preguntas también tomaron en cuenta la percepción de los docentes acerca de la repercusión e importancia de los conocimientos previos matemáticos en el momento de aprender física en el colegio o en la universidad. Además, los conocimientos que los docentes creen que deben tener los estudiantes en matemática para poder aprender física en el colegio como en la universidad.

\section{Selección de la población de estudio:}

En la primera fase o fase cualitativa, la población de estudio a la que se aplicó las entrevistas a profundidad estuvo enfocada en cinco especialistas en el área de enseñanza de la física y a cinco especialistas en la enseñanza de la Matemática, con experiencia tanto en nivel universitario como en educación diversificada. Participaron profesores de educación universitaria que habían impartido por lo menos tres veces el curso de Física General I teoría, con un grado académico de Licenciatura en Enseñanza de la Física o Física (o ramas de la física), que imparten sus lecciones en las siguientes universidades públicas: UCR, UNA y Universidad Técnica Nacional (UTN). La población de profesores de física en educación superior y en educación diversificada,que se muestreo, fue de 22 personas en ambas.

En la segunda fase se consultó a 16 estudiantes del primer curso de Física General de la UTN mediante un cuestionario, el cuál proporcionó la información de qué conocimiento matemático ellos consideran necesario para poder comprender y aprobar el curso de Física General uno. Se escogió intencionalmente a estudiantes de la UTN, debido a que esta universidad está iniciando su trayectoria y dicha información les puede dar criterio para modificar sus programas de Física Introductoria y Matemática General, para mejorar el aprendizaje de la Física de sus estudiantes. Por otra parte, se consultó a 200 alumnos distribuidos en un total de 8 secciones de Educación Diversificada, acerca de los conocimientos que ellos consideran necesarios para comprender de manera significativa los contenidos de física. Los educandos que se tomaron en cuenta para la investigación fueron aquellos que se encontraban cursando la Educación Diversificada, matemática y física, en colegios públicos, académicos, diurnos de la región central de Alajuela; dichos alumnos están en colegios que aportan estudiantes a la UTN. La muestra de escolares de Educación Diversificada se obtuvo al aplicar la fórmula estadística representada en la figura 1 , luego de una visita a todos los colegios que aportaban alumnos a los cursos de Física introductoria de la UTN:

$$
n=\left(\frac{Z_{\frac{\alpha}{2}} \sigma}{d}\right)^{2}
$$

Figura 1. Fórmula estadística aplicada para obtener la muestra poblacional representativa de los estudiantes de los colegios de secundaria. Siendo, el nivel de confianza al 95\%, $\sigma$ la desviación estándar y d, el error de muestreo del 5\%. Fuente propia.

\section{Procesamiento de la información:}

Las entrevistas fueron analizadas de forma manual mediante matrices, categorizando así los resultados lo que permitió obtener todas 
las posibles categorías de análisis, además se usó la narrativa también para la explicación de dichos resultados. Las entrevistas fueron analizadas de manera prioritaria puesto que eran la base para la elaboración de los otros instrumentos. A las respuestas obtenidas por medio de las preguntas de los cuestionarios se les aplicó previamente un análisis de componentes principales para verificar si dichos datos pueden ser correlacionables mediante una correlación de Pearson.

Los datos de las entrevistas, así como las inferencias extraídas de los cuestionarios de docentes universitarios y de secundaria fueron analizados, por medio de una triangulación de datos en matriz (Gurdián, 2007). Las opiniones presentadas por los expertos y por las otras poblaciones de estudio que fueron analizadas por medio de matrices de datos, se tomaron en cuenta únicamente los criterios en los cuales había coincidencias. Las inferencias extraídas de los cuestionarios de los estudiantes fueron triangulados con las de los cuestionarios de docentes universitarios y con la de docentes de secundaria, lo que permitió verificar datos al contrastarlos con otras fuentes para lograr la validez.

Las inferencias obtenidas fueron analizadas desde la perspectiva de los investigadores y de la teoría del aprendizaje significativo para poder ser explicadas en el contexto tratado, esto por medio de técnicas narrativas (Onwuegbuzie \& Johnson, 2006, p.55; Onwuegbuzie, Slate, Leech \& Collins, 2009, p.20).

\section{Resultados y Análisis}

Se conoce que la repercusión del conocimiento matemático para aprender física, existe debido a que hay una relación natural entre ambas disciplinas, además hay una utilidad en física, que se requiere de la matemática para establecer relaciones del entorno, agrupar fenómenos y comprenderlos y comunicarlos.

A continuación, los resultados serán explicados iniciando por los datos de especialistas en física y matemática, y posteriormente se explicará el análisis obtenido de la población estudiantes de secundaria y universidad para comprender la conclusión anterior.

\section{Especialistas en física y matemática}

Es fundamental reconocer cuáles son los elementos que intervienen con respecto al aprendizaje significativo, según el criterio de expertos. Para ello, se contestó a la pregunta: ¿cómo lograr un aprendizaje significativo de la física y la matemática?; por lo tanto, se consultó

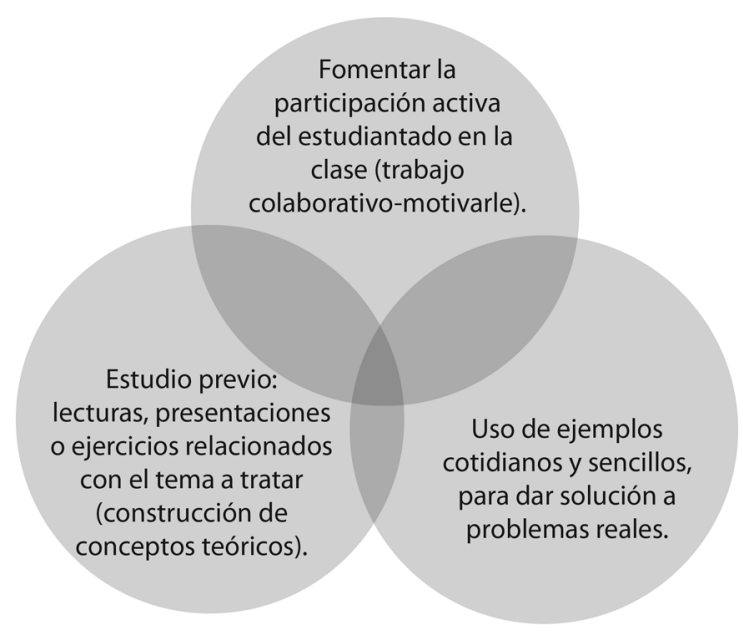

Figura 2. Estrategias didácticas concordantes con la teoría del aprendizaje significativo de acuerdo con el criterio de expertos en Física y Matemática. Elaboración propia.

a especialistas al respecto, mostrándose en la figura 2 las estrategias en las que los participantes de la entrevista coincidieron.

Ocho de los especialistas contactados, expresan que no conocen o recuerdan explícitamente la teoría del aprendizaje significativo. Pero, al explicar ellos la manera en cómo planifican o realizan sus clases, es posible evidenciar que utilizan esta teoría de aprendizaje. Como por ejemplo al tomar en cuenta elementos de la cotidianidad para explicar sus clases, construyendo el conocimiento desde el concepto más sencillo hasta alcanzar una serie de estructuras más complejas, o bien, iniciar un determinado tema desde el punto de vista intuitivo. Lo descrito por los especialistas son sin duda alguna, estrategias basadas en la teoría del aprendizaje significativo (William, 2002, p.390; Picquart, 2008, p.29). 
Desde el punto de vista anterior, todos los especialistas concuerdan en que es fundamental un excelente conocimiento matemático para aprender de manera significativa la Física de secundaria y a nivel universitario inicial. De todos los conocimientos matemáticos que ellos mencionaron hubo coincidencia total en los siguientes: aritmética, álgebra, trigonometría, geometría, teoría de funciones y geometría analítica; tanto para el colegio como para el primer curso introductorio de Física universitaria.

En el caso de secundaria, los especialistas también coincidieron en que los estudiantes de décimo año deberían dominar el uso de las potencias y la resolución de ecuaciones. Ambos contenidos muy importantes en física para trabajar con notación científica y con la resolución de problemas de movimiento rectilíneo uniforme y uniformemente acelerado, ya que son temas por tratar desde el inicio del curso lectivo.

Por otra parte, algunas de las estrategias metodológicas presentadas en la figura 2 permiten el aprendizaje por medio de la teoría de la asimilación. Esto debido a que admite la inclusión de nuevos conocimientos en la estructura cognoscitiva de la persona. (Ballesteros, Cuevas, Giraldo, Martín, Molina \& Rodríguez, 2006).

A partir de lo anterior, considerando lo expuesto por Ausubel, Novak y Hanesian (2005), se puede decir que en las edades de educación secundaria y universitaria el joven adquiere un aprendizaje por recepción; ante esto, es importante resaltar que la relación que tiene la matemática y la física según los datos recabados, va más allá que un simple cuerpo de conocimientos básicos, se trata más bien del desarrollo el pensamiento abstracto y del pensamiento lógico matemático, el cual es fundamental en la comprensión del mundo físico.

Ahora se analiza la relación entre la física y la matemática, la cual es muy estrecha. La matemática, constituye un lenguaje natural abstracto, con infinitas aplicaciones dentro de las ciencias exactas y naturales. La física, por otro lado, es la ciencia que estudia los procesos naturales, cuyo lenguaje u forma de expresar dichos procesos es mediante la Matemática. Se muestra los cuadros 1 y 2 el criterio de los expertos ante este interrogante.

Lo anterior se nota desde la formación del profesorado de secundaria en las universidades estatales.

De acuerdo con los especialistas consultados, los docentes de Matemática, aunque tienen un muy buen nivel teórico de su disciplina, no enseñan cómo relacionar la Matemática con la Física y a su vez, los docentes de Ciencias reciben una escaza formación en Matemática antes de iniciar sus cursos de Física.

Los especialistas en Física de cuarto ciclo, en Matemática de cuarto ciclo y los especialistas en Física universitaria coincidieron en los siguientes criterios: 1. La matemática es simplemente la abstracción del mundo natural,

\section{CuAdro 1}

Relación entre los conocimientos previos matemáticos y la Física,
según el criterio de los especialistas en Física. Fuente propia.

\begin{tabular}{ll}
$\begin{array}{l}\text { Criterio especialista Física } \\
\text { comparten un cuerpo de }\end{array}$ & $\begin{array}{l}\text { Relación entre conocimientos previos matemáticos y la Física } \\
\text { Debe de existir un cuerpo de conocimientos matemáticos básicos que pueda sus- } \\
\text { tentar el aprendizaje de la Física a partir de décimo año. }\end{array}$ \\
\hline $\begin{array}{l}\text { Desarrollan el pensamiento } \\
\text { Se enlazan para la enseñanza } \\
\text { de la Física }\end{array}$ & $\begin{array}{l}\text { Brinda las bases para generar el pensamiento lógico matemático } \\
\text { El lenguaje propio de la Física es la Matemática. }\end{array}$ \\
& $\begin{array}{l}\text { La Física interpreta procesos naturales y los expresa a través de estructuras } \\
\text { matemáticas. }\end{array}$
\end{tabular}




\section{CUADRO 2}

\section{Relación entre los conocimientos previos matemáticos y la Física, según el criterio de los especialistas en Física. Fuente propia.}

\begin{tabular}{ll}
\multicolumn{1}{c}{$\begin{array}{c}\text { Criterio especialista Física } \\
\text { Comparten un cuerpo de } \\
\text { conocimientos }\end{array}$} & $\begin{array}{l}\text { En cualquier circunstancia de aprendizaje, debe de haber un cuerpo de } \\
\text { conocimientos matemáticos básicos para afrontar las situaciones cotidianas. }\end{array}$ \\
& $\begin{array}{l}\text { La Física otorga a la matemática una aplicabilidad, la lleva del plano teórico } \\
\text { a la práctica. }\end{array}$ \\
& $\begin{array}{l}\text { La Matemática proporciona conocimientos procedimentales necesarios para la } \\
\text { resolución de problemas Físicos. }\end{array}$ \\
\hline $\begin{array}{l}\text { Desarrollan el pensamiento } \\
\text { Le enlazan para la matemática es fundamental ya que otorga el desarrollo del pensamiento lógico - } \\
\text { enseñanza de la Física }\end{array}$ & $\begin{array}{l}\text { La Matemático, necesario para la comprensión del mundo físico. } \\
\text { La Física no debe ser enseñada a partir de un fundamento cuantitativo o formulista. }\end{array}$ \\
& $\begin{array}{l}\text { La Física debe ser enseñada a partir del concepto físico y cotidiano y no a partir } \\
\text { de una simbología matemática, una vez comprendida la realidad Física es que se } \\
\text { construye la simbología matemática. }\end{array}$
\end{tabular}

por ello la enseñanza de la Física no debe de iniciar cuantitativamente este debe ser el paso final, y 2. La utilidad de la Matemática, es que ayuda en la comprensión de estructuras abstractas, que son usadas en Física.

Dentro del ámbito universitario, se da ya por un hecho que si el estudiante aprobó el bachillerato en secundaria, es porque de cierta forma, cuenta con todo un cuerpo de conocimientos básicos en Matemática que le permitirán sustentar el aprendizaje de la Física educación universitaria, asumiendo un pensamiento lógico matemático y abstracto bien desarrollados.

También, se infiere de los cuadros 1 y 2 , que los especialistas concuerdan que se debe enseñar de lo general a lo específico, desde lo cotidiano y no desde la simbología matemática, esto con el propósito de generar empoderamiento en el aprendizaje y que el estudiante pueda crear intuiciones deductivas e inductivas a partir de la realidad cotidiana y no del procesamiento matemático.

Por consiguiente, se puede inferir que existe una repercusión inmediata entre la necesidad de una buena base matemática y el aprendizaje de la física. Así pues, con esta investigación se demuestra cómo en Costa Rica hacen falta investigaciones que busquen la mejora en la relación entre la matemática y la física.

De acuerdo con el criterio de ambos especialistas, tal base matemática (que debe ser muy buena para que no afecte desde el punto de vista emocional el aprendizaje de la física) debe construirse y afinarse en todos los niveles educativos: desde el aula, desde la relación entre los planes de estudio, y desde la formación docente permitiendo una adecuada vinculación de los contextos y el estudiante para propiciar un adecuado aprendizaje.

Tanto la población de especialistas de física como matemática consideran que para un buen aprendizaje de conceptos y procedimientos matemáticos, no basta sólo con las estrategias de aprendizaje que se desarrollan en el aula, sino que también intervienen otros factores que se desarrollan fuera del ambiente escolar (MEP, 2007, p.5), por lo que buenas experiencias dentro y fuera del aula ayudan a promover 
el aprendizaje; y a su vez, van a ser determinantes en la asimilación y la construcción de los conceptos científicos.

\section{Profesores de secundaria y universidad}

Se consultaron 22 docentes del área de enseñanza de la Física en educación diversificada y 22 docentes del área de educación universitaria. El cuadro 3, muestra que las tres poblaciones consultadas concuerdan en que los criterios expuestos sobre los conocimientos previos matemáticos repercuten de muy buena manera en el aprendizaje significativo de la física. Se denota que existe la más alta repercusión en el segundo criterio, que es una cuestión actitudinal. Para todos los criterios no hubo diferencias significativas entre y dentro de las poblaciones de profesores de secundaria y universidad.

Tanto los docentes de secundaria como los de universidad están conscientes, que hay, dos componentes matemáticos fundamentales para aprender física: a) el componente procedimental de la matemática (traducción del mundo físico al lenguaje matemático), b) el componente del desarrollo del pensamiento. Su desarrollo previo es fundamental para que se dé el aprendizaje significativo de la física.

Malas bases en los conocimientos previos en matemática y poco pensamiento matemático hacen que el estudiante no logre inferir secuencias, tendencias o no logre agrupar eventos similares o no los logra agrupar a través de ecuaciones matemáticas; esto conlleva a que el aprendizaje de la Física sea una labor mucho más difícil.

Se resalta como pieza fundamental de la investigación que se reconozca cual es la repercusión de los conocimientos matemáticos en el aprendizaje de la física, para tal fin, se trianguló la opinión de los tres grupos de investigación consultados y la tabla 2 refleja el criterio de cada población.

El cuadro 4, demuestra que las tres poblaciones consideran que hay conceptos

\section{CuAdRo 3}

\section{Matriz de la triangulación sobre diferentes criterios de repercusión que tiene los conocimientos previos matemáticos en el aprendizaje de la Física, según Especialistas, Docentes de Secundaria y Profesores de universidad}

\begin{tabular}{|c|c|c|}
\hline Criterio especialistas & Profesores de secundaria & Profesores de universidad \\
\hline $\begin{array}{l}\text { El pensamiento matemático potencial } \\
\text { a su vez el pensamiento hacia la física. }\end{array}$ & $\begin{array}{l}10 \text { de los docentes consultados, } \\
\text { opina que esta categoría tiene } \\
\text { buena repercusión en los } \\
\text { conocimientos matemáticos. }\end{array}$ & $\begin{array}{l}10 \text { de los docentes consultados, } \\
\text { opina que esta categoría tiene } \\
\text { buena repercusión en los } \\
\text { conocimientos matemáticos. }\end{array}$ \\
\hline
\end{tabular}

Permite realizar una correcta $\quad 11$ docentes consideraron que 12 docentes consideraron que
traducción del mundo natural a un lenguaje universal (matemática).

esta categoría posee la más alta repercusión en los conocimientos matemáticos 4 docentes consideraron que la Matemática ayuda a comprender fenómenos físicos.

\begin{tabular}{|c|c|c|}
\hline $\begin{array}{l}\text { Una buena base matemática } \\
\text { garantiza el éxito y la motivación } \\
\text { al aprender Física }\end{array}$ & $\begin{array}{l}10 \text { docentes opinaron que esta } \\
\text { categoría tiene buena repercusión. }\end{array}$ & $\begin{array}{l}12 \text { docentes opinaron que esta } \\
\text { categoría tiene buena repercusión } \\
\text { en los conocimientos matemáticos. }\end{array}$ \\
\hline $\begin{array}{l}\text { El conocimiento matemático } \\
\text { permite que el estudiante establezca } \\
\text { relaciones, agrupaciones y tendencias } \\
\text { del mundo físico. }\end{array}$ & $\begin{array}{l}9 \text { docentes opinaron que esta } \\
\text { categoría tiene buena repercusión } \\
\text { en los conocimientos matemáticos. } \\
\text { La matemática brinda principios } \\
\text { básicos para aprender física. }\end{array}$ & $\begin{array}{l}12 \text { docentes consideraron que } \\
\text { la repercusión es directa, la } \\
\text { matemática provee habilidades } \\
\text { procedimentales esenciales para la } \\
\text { resolución de problemas físicos. }\end{array}$ \\
\hline
\end{tabular} esta categoría posee la más alta repercusión en los conocimientos matemáticos.

.

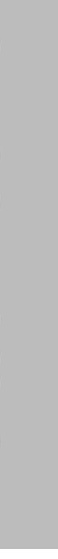


CUADRO 4

Matriz de la triangulación sobre la repercusión en el aprendizaje de la física que presentan los conocimientos previos matemáticos, según los tres grupos de investigación.

\begin{tabular}{|c|c|c|}
\hline Estudiantes de Colegio y Universidad & $\begin{array}{c}\text { Profesores de Colegio y } \\
\text { Universidad }\end{array}$ & Especialistas \\
\hline $\begin{array}{l}\text { Hay cosas básicas de la Matemática que sirven a la hora } \\
\text { de estudiar Física. } \\
\text { Esa repercusión se siente en la universidad, ya que } \\
\text { si se traen malas bases del colegio imposibilitan el } \\
\text { aprendizaje. } \\
\text { Hay algunos conocimientos previos de Matemática } \\
\text { que ayudan. }\end{array}$ & $\begin{array}{l}\text { La Matemática brinda } \\
\text { principios básicos de } \\
\text { conocimientos para } \\
\text { aprender física. }\end{array}$ & $\begin{array}{l}\text { El conocimiento matemático } \\
\text { permite que el estudiante } \\
\text { establezca relaciones, } \\
\text { agrupaciones y tendencias del } \\
\text { mundo físico. }\end{array}$ \\
\hline $\begin{array}{l}\text { La Matemática sirve para el análisis y comprensión de } \\
\text { los problemas en Física. } \\
\text { La Matemática facilita los procesos en Física. } \\
\text { Muchas veces la Física ayuda a comprender como } \\
\text { resolver ejercicios en Matemática. } \\
\text { La Física ayuda a comprender las reglas matemáticas. } \\
\text { La Física le otorga "aplicabilidad" a la matemática. La } \\
\text { matemática y la física van de la mano. }\end{array}$ & $\begin{array}{l}\text { La Matemática } \\
\text { ayuda a comprender } \\
\text { fenómenos físicos. } \\
\text { La matemática ayuda } \\
\text { a interpretar datos } \\
\text { en física. }\end{array}$ & $\begin{array}{l}\text { La repercusión es directa, la } \\
\text { matemática provee habilidades } \\
\text { procedimentales esenciales } \\
\text { para la resolución de problemas } \\
\text { físicos. } \\
\text { Cualquier actividad que potencia } \\
\text { el pensamiento matemático } \\
\text { potenciará el pensamiento físico. } \\
\text { Modelación del mundo físico en } \\
\text { un lenguaje universal. }\end{array}$ \\
\hline
\end{tabular}

y procedimientos matemáticos fundamentales, que se deben saber antes de iniciar el aprendizaje de la física. Algo importante es la opinión de los estudiantes que indican estar conscientes de que, sí no poseen buenos conocimientos previos matemáticos se les puede dificultar el aprendizaje de la física.

Los vacíos en los conocimientos previos matemáticos se llegan a expresar hasta en el primer curso de Física de la universidad, y son los docentes universitarios y los especialistas los que han recalcado que cuando un estudiante matricula un curso de Física introductorio, parten del hecho de que ya posee conceptos matemáticos básicos adquiridos, cuestión que no siempre es así. Esta situación se podría considerar como un factor asociado al fracaso académico.

En la teoría del aprendizaje significativo, la introducción de un concepto responde a un proceso de discusión y reelaboración a partir de una problemática concreta (lo que requiere que el docente tenga claro el panorama de lo que se demanda como perfil del estudiante que ingresa y egresa según los programas nacionales de estudio) (MEP, 2007, p. 6). Ante dicho panorama, es fundamental saber que la matemática y la física son disciplinas que se nutren una de la otra, por lo que se debe tener fundamento de ambas para lograr el aprendizaje deseado.

De acuerdo con lo dicho anteriormente, los conceptos matemáticos, no son exclusivamente de dominio de la matemática, sino que estos conceptos también son comunes en otras áreas del conocimiento, como lo plantean Cantoral y Farfán (2003), citados por Cordero (2005), en la educación superior es común que otras disciplinas del conocimiento como Química, Física, Ingenierías, Economía, etc, utilicen los conceptos matemáticos.

\section{Estudiantes de secundaria y universidad}

Se procedió a aplicar un cuestionario a colegiales de educación diversificada del área 
de Alajuela que pueden aportan estudiantes a la Universidad Técnica Nacional (UTN). Se aplicaron 200 cuestionarios, a estudiantes de los colegios públicos académicos siguientes: Instituto de Alajuela, Colegio Redentorista San Alfonso, Colegio del Carmen, Liceo de la Guácima, Colegio Ambientalista del Roble, Liceo de Poás, Liceo de Tuetal Norte y Liceo Otilio Ulate Blanco. Los resultados obtenidos se resumen a continuación.

En el caso de los estudiantes de secundaria y universidad, se obtienen las siguientes percepciones para la pregunta abierta: ¿Tiene alguna repercusión en su aprendizaje de la Física, todos los conocimientos previos matemáticos que usted ha aprendido? Para explicar los resultados obtenidos a esta interrogante se muestra la siguiente figura 3 , en donde se denotan los grados de repercusión valorados para este grupo.

De la figura 3, se infiere que 12 estudiantes universitarios consideraron que si repercute. No obstante, estadísticamente hablando no se puede decir que esa diferencia sea significativa. ( $\mathrm{K}-$ $\mathrm{W}=0,02 ; \mathrm{p}>0,05)$. En el caso de los estudiantes de colegio, 136 estudiantes de los 200 consultados, respondieron que sí repercute, 26 de ellos consideraron que no mientras que 9 de ellos pensaron que más o menos. Sin embargo no se puede decir que esas diferencias sean significativas $(\mathrm{K}-\mathrm{W}=0,333 ; \mathrm{p}>0,05)$.
También es de recalcar que 7 de los 16 estudiantes de educación universitaria también consideran que sí repercuten los conocimientos previos matemáticos; y todos reconocen que existe una total o mucha repercusión de esos conocimientos sobre el aprendizaje de la Física. Solo tres de ellos consideraron que no había relación entre la matemática y la Física; en este caso sí hay una diferencia significativa estadística en el pensamiento de los estudiantes de universidad $(\mathrm{K}-\mathrm{W}=0,02 ; \mathrm{p}<0,05)$.

Estas posiciones de los estudiantes son fundamentales, ya que definitivamente en ellos hay conciencia de que las bases matemáticas repercuten directamente en el aprendizaje de la física, los estudiantes universitarios por su nivel de desarrollo presentan una mayor responsabilidad en su aprendizaje de lo que se podría prever sus respuestas, lo que se observa también en la tendencia numérica; por lo tanto, se puede decir que ellos piensan que los conocimientos previos matemáticos si repercuten significativamente $\mathrm{y}$ mucho ( $\mathrm{K}-\mathrm{W}=6,55 ; \mathrm{p}<0,05)$. Esto lo justifican con frases tales como:

"Conocimientos de tipo práctico de la matemática que ayudan a comprender la Física, esa repercusión se siente en la universidad, ya que se traen malas bases del colegio que imposibilitan aprender fisica".

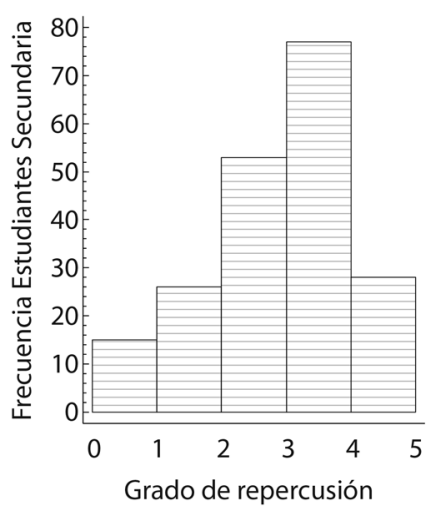

Significado de los valores de grado de concordancia a lo expresado en el enunicado:

1: no hay repercusión 2: poca repercusión 3: mediana repercusión 4: mucha repercusión 5: repercusión total.

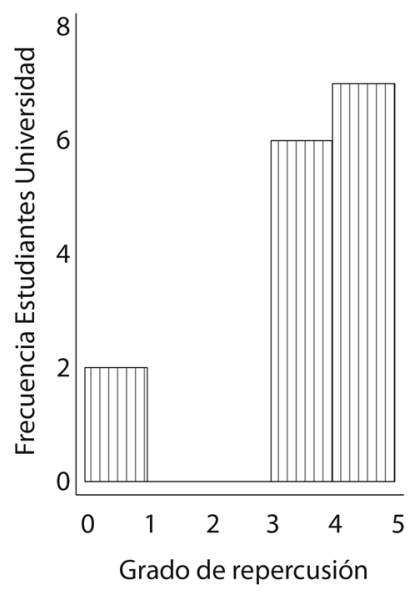

Figura 3. Opinión de los estudiantes de colegio como de universidad sobre el grado de la repercusión entre los conocimientos previos matemáticos y el aprendizaje de la Física. Fuente Propia. 


\section{CUADRO 5}

Triangulación de datos sobre la repercusión de los conocimientos matemáticos previos a la hora de aprender Física, según estudiantes de secundaria, universitaria y especialistas.

\begin{tabular}{|c|c|}
\hline Estudiantes de Colegio & Estudiantes de Universidad \\
\hline $\begin{array}{l}\text { Hay cosas básicas de la matemática que } \\
\text { sirven a la hora de estudiar Física. }\end{array}$ & $\begin{array}{l}\text { Esa repercusión se siente en } \\
\text { la universidad, ya que si se } \\
\text { traen malas bases del colegio }\end{array}$ \\
\hline $\begin{array}{l}\text { Hay algunos conocimientos previos de } \\
\text { Matemática que ayudan. }\end{array}$ & imposibilitan en aprendizaje. \\
\hline $\begin{array}{l}\text { La Matemática sirve para el análisis y } \\
\text { comprensión de los problemas en Físic }\end{array}$ & $\begin{array}{l}\text { Conocimientos de tipo práctico } \\
\text { de la Matemática ayudan a } \\
\text { comprender la Física. }\end{array}$ \\
\hline
\end{tabular}

El conocimiento matemático permite que el estudiante establezca relaciones, agrupaciones y tendencias del mundo físico.

La repercusión es directa, la matemática provee habilidades procedimentales esenciales para la resolución de problemas Físicos. Cualquier actividad que potencia el pensamiento matemático potenciará el pensamiento físico.

La Matemática y la Física van de la mano.
Modelación del mundo Físico en un lenguaje universal.

La Matemáti
en Física.

Muchas veces la Física ayuda a

comprender como resolver ejercicios

en Matemática.

La Física ayuda a comprender las reglas

Matemáticas.

La Física le otorga "aplicabilidad"

a la Matemática.

La repercusión es negativa ya que muchas No expresaron opiniones

veces la materia de ambas se enreda.

No es primordial saber matemática para aprender Física.
El éxito y buenas bases matemáticas garantizan seguridad y una actitud positiva hacia la Física.
A su vez el cuadro 5, indica una serie de opiniones brindadas por los estudiantes que fueron contrastadas con la de los especialistas, y se muestra que las repercusiones tienen una coherencia horizontal, agrupadas en tres grandes grupos; las dos primeras de esas categorías corresponden a repercusiones del tipo conceptual y del tipo procedimental, la tercera categoría corresponde a repercusiones del tipo actitudinal fuertemente influenciadas por las otras dos.

Se puede ver cómo las buenas bases matemáticas garantizan el éxito y una comprensión de la Física que se ve reflejado en una buena actitud hacia ambas disciplinas. A su vez se observa el grado de conciencia y responsabilidad de los estudiantes, además, cómo ellos ven que buenas bases matemáticas, puede ayudar a solucionar de varias formas un mismo problema físico. $\mathrm{O}$ sea, un buen desarrollo del pensamiento y procedimientos matemáticos garantizan maneras de abordar un problema físico y darle solución.

Hay que recalcar que en caso de estudiantes de colegio, en ninguna de las categorías hubo más de 30 estudiantes de la población total de doscientos, que estuvieron en desacuerdo con dichas opiniones, y en el caso de los estudiantes universitarios no estuvieron en desacuerdo en dichas opiniones.

Debido a que la muestra de estudiantes de universidad es pequeña se procedió a realizar 
una serie de correlaciones para determinar si algunas de las opiniones brindadas por el estudiantado se pueden relacionar con otras.

Ahora establecida dicha repercusión, se puede trabajar sobre qué cuerpo de conocimientos consideran los estudiantes universitarios se deben tener para el aprendizaje de la Física universitaria, los cuales se detallan en el cuadro 6 .

Se muestra otra vez la coincidencia en el Algebra, Trigonometría, Funciones, Aritmética, conocimientos que se podría decir son adquiridos en el colegio y se recalca otra vez el uso del Cálculo como conocimientos previos para el aprendizaje significativo de la Física universitaria.

Es importante reconocer los conocimientos matemáticos que se resaltan como necesarios para aprender Física a nivel universitario, tener presente que el docente debe al iniciar la enseñanza de un nuevo conocimiento partir de lo que el estudiante ya sabe; por tanto para el proceso de enseñanza hay que tener en cuenta lo que el estudiante conoce (preconcepciones o conceptos previos) y de las dificultades que presentan los estudiantes (Cravino \& Lopes, 2003, p.475; Chacón, 2008, p. 134; Picquart, 2008, p.30 \& Rodríguez, 2009, p.62) .

Es importante reconocer que si se empieza aprender la Física sin el pensamiento lógico matemático bien desarrollado o la falta de procedimientos matemáticos, conllevará una mala experiencia en el aprendizaje de la Física, ya que impide desarrollar adecuadamente el entendimiento de los procesos para solucionar un problema. Por lo tanto estas malas experiencias se ven reflejadas en los comentarios que brindaron los estudiantes de colegio sobre repercusiones negativas o que perciban a la Matemática y la Física que no deben de relacionarse o no tienen nada que ver una con la otra (Rozende \& Ostermann, 2006,p. 390).

Hay un grado de metacognición en las dos poblaciones de estudiantes, ambos en mayoría creen que hay una gran repercusión de los conocimientos previos matemáticos a la hora de aprender significativamente Física. Con expresiones lo demuestran: "malas bases de Matemática en el colegio después se resienten en Física I", en el caso de estudiantes universitarios o frases de los estudiantes de colegio como: "La matemática ayuda al análisis y comprensión de los problemas en Física".

Ayuda a concordar con aspectos mencionados por los especialistas y secundados por los docentes: el desarrollo del pensamiento matemático es primordial para el aprendizaje

\section{CUADRO 6 \\ Conocimientos matemáticos previos que según los estudiantes universitarios necesitan para aprender Física a nivel universitario. En orden de importancia según los mismos estudiantes.}

Conocimientos matemáticos

Algebra

Aritmética básica

Trigonometría

Cálculo

Funciones

significativo de la Física, así como procedimientos previos ya adquiridos son esenciales, definitivamente deben ser tomados en cuentan como puntos clave en la planificación del docente de Física tanto en la universidad como en el colegio.

\section{Conclusiones}

Entrelazar los conocimientos previos matemáticos con los nuevos conocimientos físicos a hacer asimilados es importantísimo para lograr el aprendizaje significativo. Por ello es esencial, la articulación a nivel de programas es fundamental para logra la construcción del conocimiento, tal y como lo plantean la fundamentación de dichos programas.

Claro está, los estudiantes no saben de tipos de pensamientos o procedimientos o conceptos matemáticos o de conceptos físicos, pero a la hora de analizar sus respuestas con respecto a las opiniones dadas por los especialistas ellos sí pueden discernir que algunos conceptos y procedimientos aprendidos en matemática son útiles al estudiar física; que la tendencias o relaciones de la realidad pueden describirse 
por ecuaciones matemáticas; en la utilidad de la matemática al aprender física expresan: un problema físico se puede abordar de varias maneras con la matemática.

Cabe mencionar que ellos también conocen la dimensión actitudinal y quizás muchas de sus respuestas o comentarios negativos van impregnadas por ese factor que no se puede ni negar ni ocultar, malos resultados en Matemática afectarán el aprendizaje de la Física, desde la parte emocional y la parte de faltante de conocimientos previos.

De manera final, se sabe que la Matemática tiene una relación innegable con la Física, ésta es la forma por el cual, el medio físico se traduce, es a través del lenguaje matemático que se pueden representar los conceptos físicos y establecerse relaciones y tendencias. Además, todas las poblaciones analizadas: especialistas, docentes de colegio y universidad y los estudiantes consideran vital que se tenga dominio de los conocimientos previos matemáticos para abordar el aprendizaje de la Física. Por ello, los conocimientos previos matemáticos si repercuten en el aprendizaje significativo de la Física y son importantes a nivel del pensamiento, a nivel de procedimientos e incluso a nivel actitudinal.

\section{Referencias}

Alfaro, G. \& Villegas, L. 2010. Tercer Informe del Estado de la Educación. La Educación Científica en Costa Rica. San José, Costa Rica. CONARE. (Informe de Estado)

Ausubel, D., Novak, J. \& Hanesian, H. 2005. Psicología Educativa, Un Punto de vista Cognoscitivo. México DF, México. Editorial Trillas. 100p. (Libro)

Ballesteros, A., Cuevas, C., Giraldo, L., Martín, I., Molina, A., Rodríguez, A. \& Velez, U. 2006. Mapas conceptuales: Una técnica para aprender. Madrid, España. Editorial Narcea.15p.(Libro)

Chacón, C. 2008. Problemas Fundamentales de la Formación en Física Básica. TEA, 24. (Revista)

Chang, W. \& Bell, B. 2002. Making Content Easier or Adding More Challenge in Year One University Physics? Research in Science Education, 32.(Revista)
Cordero, F. 2005. El Rol de Algunas Categorías del Conocimiento Matemático de Orden Superior. Una Socioepistemología de la Integral. RELIME, 8(3). (Revista)

Cravino, J. \& Lopes, B. 2003. La Enseñanza de Física General en la Universidad. Propuestas de Investigación. Enseñanza de las Ciencias, 21(3). (Revista)

Gurdián - Fernández, L. 2007. El Paradigma Cualitativo en la Investigación Socio - Educativa. San José, Costa Rica. Editorial Colección IDER. 150p.(Libro)

Hernández, R., Fernández - Collado, C. \& Batista, P. 2008. Metodología de la Investigación. México D.F, Mexico. Editorial Mc Graw Hill. 150p. (Libro)

Ministerio de Educación Pública. 2005 - 2012. Programas de Estudio de Matemática. MEP. San José, Costa Rica. (Programa de Estudio)

Onwuegbuzie, A. \& Johnson, R. 2006. The Validity Issue in Mixed Research. Research in the Schools 13(1). (Revista)

Onwuegbuzie, A., Slate, J., Leech, N., \& Collins, K. 2009. Mixed Data Analysis: Advanced Integration Techniques. Internacional Journal of Multiple Research Approaches, 3. (Revista).

Owen, S., Dickson, D., Stanisstrest, M., \& Boyes, E. 2008. Teaching physics: Students' attitudes towards different learning activities. Research in Science and Technological Education, 26(2). (Revista).

Picquart. M., 2008. ¿Qué podemos hacer para lograr un aprendizaje significativo de la Física? Latin American Journalist Physics Education 2(1). (Revista).

Rodríguez, J. 2009. Cambios Metodológicos Relacionados con el Aprendizaje de las Ciencias. Revista Educación, 33 (1). (Revista)

Román, I y Vargas, J. 2015. Quinto Informe Estado de la Educación en Costa Rica. San Jose: Programa Estado de la Nación. (Informe de Estado)

Rossana, C. Di Domenicantonio, M, Prodanoff, F. Tolosa, E. \& Guarepi, V. 2006. Acciones Interdisciplinarias entre la Matemática y la Física para Mejorar la Enseñanza del Cálculo Vectorial. Facultad de Ingenierías, Universidad Nacional de la Plata Argentina. Recuperado de: https://www.academia. edu/934032/Acciones_interdisciplinarias_entre_ 
matematica_y_fisica_para_mejorar_la_enseñanza_y_ aprendizaje_del_calculo_vectorial. (Artículo en línea)

Rozende, F. \& Ostermann, F. 2006. Enseñanza-aprendizaje de la física en Brasil: confrontando teoría y práctica en el inicio del siglo XXI. Enseñanza de las Ciencias 24(3). (Revista)

Salinas, J., Cudmani, L., \& Pesa, M. 1996. Modos Espontáneos de Razonar: Un Análisis de su Incidencia sobre el Aprendizaje del conocimiento Físico a Nivel
Universitario Básico. Enseñanza de las Ciencias, 14(2). (Revista)

Seas, J. (2015). Didáctica General I. San José, Costa Rica. Editorial EUNED. 100p. (Libro)

William, L., W. Gerace, \& Robert, J. 2002. Resolución de problemas basado en el análisis. Hacer del análisis y razonamiento el foco de la Enseñanza de la Física. Enseñanza de las Ciencias, 20(3). (Revista) 
